\title{
Reducing binge drinking in adolescent males
}

\author{
Facing up to binge drinking: reducing binge drinking in adolescent males \\ M. Dempster, G. Newell, G. Cowan and J. Marley Br Dent J 2006; 201: 587-590
}

\section{Objective}

To evaluate the effectiveness of an orofacial trauma-based brief intervention, designed to raise adolescent males' awareness about the immediate dangers of binge drinking.

Design

Non-randomised controlled exploratory trial.

Setting

Secondary level schools.

\section{Materials \&t methods}

Pre, post and follow up validated questionnaires were used to assess a variety of descriptive data and changes in behaviour. Sixty Year 12 students were recruited in the pilot study and 182 in the definitive study. Intervention

A brief visual presentation containing salient information and anonymised photographs relating to orofacial injuries.

Main outcome measures

Intention to binge drink.

Results

The majority of participants obtained alcohol from off-license or licensed premises. At the commencement of the study, $68 \%$ of the participants were regular drinkers. Whilst there was no change in drinking behaviour, the intervention group reported that it was significantly more likely (compared to the control group) that they would reduce their drinking to less than binge levels.

Conclusion

The intervention resulted in participants reporting a more negative attitude towards binge drinking and increased their intention to disengage from binge drinking.

\section{IN BRIEF}

- Attitudes to binge drinking among adolescent males are difficult to change using traditional health promotion messages.

- This paper demonstrates that an orofacial trauma-based brief intervention can change attitudes to binge drinking among adolescent males, but not drinking behaviour.

- An orofacial trauma-based brief intervention could be the first step of a larger intervention which may reduce binge drinking among adolescent males.

\section{COMMENT}

Because we live in an age in which education is seen as the solution to most major problems in society, it is easy to assume that all education must be effective. However, we know from high quality evaluations of such initiatives as early driver training, sex education, and the 'Scared Straight' initiative to deter adolescents from offending, that education in these areas during the secondary school years often makes things worse. This makes this exploratory trial very welcome: we have to know that secondary school efforts to tackle alcohol misuse are effective before investing time in them - especially the time of health professionals who might achieve more in their clinics.

The results suggest there might be some benefit in terms of changing attitudes to binge drinking among secondary school children but also that drinking itself may be unaffected by this intervention.

Alcohol misuse can cause a range of oral disease, most importantly mouth cancer. Intervening in the lives of adolescents who binge drink but have yet to become dependent is a very attractive strategy.

There are at least three meta-analyses which demonstrate the benefit of motivational interviewing as a treatment for alcohol misuse. ${ }^{1-3}$ This approach is most effective if it capitalises on a 'teachable moment' represented by, for example, suture removal in a trauma clinic in the few days following a facial laceration sustained whilst intoxicated.

Interestingly, tackling alcohol misuse seems most likely to reduce injury in violence by reducing vulnerability to injury rather than by reducing the propensity to act violently. For example, a recent randomised trial of alcohol misuse intervention in offenders demonstrated a treatment effect in terms of reducing offenders' injury rates but not offending rates. ${ }^{4}$

I hope that the authors will apply the lessons they have learnt in a formal controlled trial designed to capitalise on teachable moments in the lives of young people.

J. Shepherd, Professor of Oral and Maxillofacial Surgery, Wales College of Medicine, Cardiff University, Cardiff

1. Effective Health Care Research Team. Brief interventions and alcohol use. Are brief interventions effective in reducing harm associated with alcohol consumption? Effective Health Care Bulletin 1993; 7: 1-13.

2. Mattick $R$, Jarvis T. Brief or minimal intervention for 'alcoholics.' The evidence suggests otherwise. Drug Alcohol Rev 1994; 13: 137-144.

3. Wilk A, Jensen $V$, Havighurst T. Meta-analysis of randomised controlled trials addressing brief interventions in heavy alcohol drinkers. J Gen Intern Med 1997 12: 274-283.

4. Watt K Shepherd J P. A randomised controlled trial of an alcohol brief intervention for violent offenders. Cardiff: Wales Office for Research in Health and Social Care, 2005.

DOI: $10.1038 / s j . b d j .4814213$ 